\title{
III.-Note on the Constitution of Carbonic Oxide.
}

\section{By J. Alfred Wanklyn.}

The general result of modern chemical research bas been the addition of a new clause to Dalton's atomic theory. To the dictum "The elements consist of atoms of different weights, and the compounds are simple groupings of these atoms," we make this modern addition : An atom of an element is invariably combined with the same number of equivalents. The apparent contradictions of the law, which occur very frequently, are explained by self-saturation, more or less complete, as the case may be. In the compounds of hydrogen with carbon, which but a few years ago seemed to defy all attempts at classification, we have an admirable example of the working of the law. We find the utmost diversity in the ratio between the hydrogen and the carbon, but the state of condensation of each hydrocarbon always falls out so as to admit of the appropriate amount of self-saturation in every case ; and, indeed, organic chemistry, as a whole, teaches this doctrine of uniform saturation. Inorganic chemistry, on the other hand, abounds in examples of failure of the law.

Proto- and per-salts of the metals (though these are easily explicable), ammonia and hydrochlorate of ammonia, the oxides of nitrogen, and oxide of carbon, are cited against the law.

In presence of these instances chemists take very different views.

One class of chemists abandon the greater part of the law, and insist only upon the declaration that the atoms of one set of elements combine with an even number of equivalents, whilst the atoms of the other set of elements combine with an uneven number of equivalents. These chemists are satisfied with very little predication in any given case, and yet have not disposed of all of the adverse cases, for the oxides of nitrogen do not fall under even this very limited statement of the law.

A second class of chemists take refuge in the possibility of there being two orders of chemical combination, the one atomic, the other mere apposition. In the hands of these chemists the law is in danger of being reduced to a mere figure of speech.

A third class abide by the law in its integrity, and look forward to au explanation of the inorganic difficulties. 
I have to offer an explanation of the anomaly which appears in the constitution of oxide of carbon. This compound contains only one atom of carbon in its molecule, and yet the one atom of carbon is united with only two equiralents of oxygen. It is unrepresented by either a hydrogen, or a chlorine, or even a sulphur analogue, for methylene, chloro-methylene, and protosulphide of carbon have not yet been obtained, though they have often been sought. This circumstance points to the oxygen as the source of the irregularity. Let us put 8 for the atomic weight of oxygen, and let the atom of oxygen be capable of saturating two atoms of hydrogen, and the difficulty with regard to carbonic oxide vanishes.

$$
\mathrm{O}^{\prime \prime}=8 \text {. }
$$

Marsh Gas. Carbonic Oxide. Carbonic Acid. C $\mid \begin{aligned} & \mathrm{H} \\ & \mathrm{H} \\ & \mathrm{H} \\ & \mathrm{H}\end{aligned}$

C $\mid \begin{aligned} & \mathrm{O}^{\prime \prime} \\ & \mathrm{O}^{\prime \prime}\end{aligned}$

$\mathrm{C} \mid \begin{aligned} & \mathrm{O}_{2}^{\prime \prime} \\ & \mathrm{O}_{2}^{\prime \prime}\end{aligned}$

This being so, the usual oxygen-compounds such as water, carbonic acid, the common oxides of the metals will contain oxygen in its second state of condensation. Now, there is no reason for believing that the commonest state of combination of oxygen, or any other polyatomic element, is of necessity its simplest state. The compounds of carbon have taught us how very common and stable a complex grouping of an element may be; and too little is known about the various states of condensation of carbon, to admit of our attaching much weight to the circumstance that compounds containing only 8 of oxygen in the molecule are as yet unrecognised ; and, moreover, $R$ ose's quadrantoxides appear to offer examples of the occurrence of this simple oxygen-atom in the uncondensed state. How, for example, is the quadrantoxide of the monatomic metal, silver (containing 4 of oxygen to 108 of silver) to be explained, if the atomic weight of oxygen be not 8 ?

I will conclude with suggesting a nomenclature and a notation to express the different states of condensation of a given element, taking carbon as the example. The carbon of marsh-gas I propose to call " one-fold carbon," or carbon simplex (symbol C). The carbon of alcohol and acetic acid, two-fold carbon, or carbon duplex (symbol E); the carbon of glycerin, three-fold 
DEBOS ON THE CONSTITUTION, ETC.

carbon," or carbon triplex (symbol E), \&c. ; and carbon multiplex is a general expression for all but carbon simplex.

The following are examples of this notation ( $I$ have taken oxygen simplex $\mathrm{O}^{\prime \prime}=8$ ) :-

Alcohol $\ldots \ldots \ldots \ldots \ldots \ldots \quad \mathrm{EH}_{6}$ (2)

Methylic Ether......... $\mathrm{C}_{2} \mathrm{H}_{6}{ }^{\text {(2) }}$

Tetrylic Alcohol $\ldots \ldots \ldots, \mathbf{C H}_{10}^{(2)}$

Common Ether ......... ${ }_{2}^{2} \mathrm{H}_{10}$ (อ) 